\title{
Análisis de la motivación, participación y actitud en actividad física extraescolar Analysis of the motivation, attitude and participation in extracurricular physical activity
}

\author{
*José Arturo Abraldes Valeiras, **Francisco M. Argudo Iturriaga \\ *Universidad Católica San Antonio (España), *Universidad Autónoma de Madrid (España)
}

\begin{abstract}
Resumen: El objetivo de este estudio fue evaluar el efecto de los estilos de enseñanza sobre la participación y motivación en el alumnado de Educación Física de $3^{\circ}$ de la E.S.O. Se realizó un diseño cuasi-experimental a un grupo de 30 alumnos, dividiéndolo en dos subgrupos, Ay B, de 15 alumnos, a los que se les aplicará el estilo de enseñanza tradicional (de tipo mando directo) al subgrupo A, y un estilo que fomente la participación al subgrupo B. El estudio experimental abarcó un total de 9 sesiones prácticas de Educación Física en un Centro de Educación Secundaria Obligatoria. Se pasó un cuestionario de actitud hacia la actividad física antes de comenzar y al finalizar la unidad didáctica de juegos y deportes, para valorar si se produce algún tipo de modificación en la conducta de los alumnos; y un cuestionario de satisfacción-motivación al término de cada bloque de sesiones. Las variables objeto del estudio serán: los estilos de enseñanza tradicionales de mando directo y estilos que favorecen la socialización, la motivación, la participación, la actitud y la satisfacción.
\end{abstract}

Palabras clave: Estilos de enseñanza, motivación, participación, actividad física extraescolar.

Abstract: The current essay tries to evaluate the effect of the styles of teaching about participation and motivation among the students of Physical Education in the third year of secondary education. A quasi-experimental design was carried out in a group of 30 students, and it was divided into two subgroups, A and B, of 15 students, to which it will be applied the traditional teaching style (of direct lead character) to the subgroup A, and a style that will promote the participation of the subgroup B. The experimental essay covered a total of 9 practical sessions of Physical Education in a school of secondary education. An attitude questionnaire towards physical activity was done before starting and finishing the educational unit of games and sports, to value if any kind of modification was produced in the behaviour of the students; and a satisfaction-motivation questionnaire at the end of each section of sessions. Variables subject of the study will be: traditional teaching style of direct lead and styles that help to socialization, motivation, participation, attitude and satisfaction.

Key words: teaching styles, motivation, participation, out-of-school physical activity.

\section{Introducción}

Los elevados índices de sedentarismo del mundo occidental, el aumento de las enfermedades cardiovasculares, del sobrepeso y la pérdida de condición física y funcional de la población, ya no es sólo un problema del adulto sino también de la población joven, que carece del interés suficiente por el ejercicio físico.

La disponibilidad de tiempo libre es un factor determinante para la posible realización de actividad física, e incluso para el planteamiento de ésta. Los espacios para el movimiento de los niños se han visto reducidos por acción del modernismo, y el deporte escolar no ha podido dar respuesta ni generar motivación en relación al deporte y la recreación.

Gran parte de la carencia del interés para la práctica de ejercicio, se manifiesta de forma significativa en los centros de enseñanza, en los que los alumnos no se encuentran lo suficientemente motivados, llegando esto a ser una importante causa justificada del abandono de la actividad física. En este sentido, Ruiz y García (2002) afirman que «las mayores tasas de práctica fisico-deportiva se producen entre los más jóvenes, disminuyendo al incrementarse la edad».

Se puede observar en los estudios realizados por el Centro de Investigaciones Sociológicas (García, 2005) que la práctica deportiva aumenta en edades comprendidas entre los 25 y 44 años, lo que demuestra con el apoyo de otros estudios (Gómez, Ruiz, García, y Piéron, 2002), que la población con edades comprendidas entre los 16 y 20 años abandonan la realización de actividades físicas por la falta de tiempo libre, considerando éste poco tiempo en los días laborables (menos de dos horas libres), y normal en los fines de semana.

La motivación hacia la práctica físico-deportiva constituye un elemento de vital importancia en el proceso de enseñanza/aprendizaje. Florence (1991) señala que el alumno motivado se manifiesta en el grupo de clase con diferentes conductas: es activo, se agota, atiende a las explicaciones, las cuestiona, ayuda a los compañeros, se interesa por

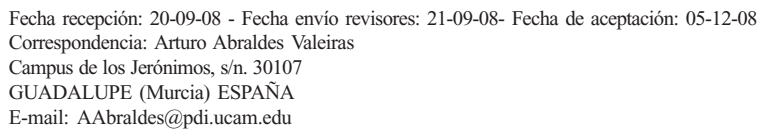

Fecha recepción: 20-09-08 - Fecha envío revisores: 21-09-08- Fecha de aceptación: 05-12-08 E-mail: AAbraldes@pdi.ucam.edu

hacer más, adelanta a su turno, está alegre, se interesa por su desempeño, repite sus ejercicios y se entrena fuera del curso. En suma, siente placer y deseo de ejercitarse y aprender en la clase de Educación Física.

Esta motivación actúa de forma diferente según se trate de motivación intrínseca o motivación extrínseca. La motivación intrínseca proporciona diversión, un sentimiento de competencia, habilidad o autorrealización, lo que da lugar a una mayor entrega y persistencia de la actividad; por otro lado la motivación extrínseca se encarga de las recompensas externas de cualquier tipo que conlleve la participación, especialmente cuando es exitosa.

La aportación de los docentes de Educación Física y sus métodos o técnicas de enseñanza tienen mucho que ver en la falta de motivación de los alumnos. Según Bernardo (1991) «el fracaso de muchos educadores radica en que no motivan convenientemente en sus clases» (citado en Sáenz, Ibáñezy Jiménez, 2000). Para Gallego (1990) «la motivación es una de las preocupaciones más grandes que tienen actualmente los profesores principiantes») (citado en Sáenz et al., 2000).

A su vez, los estilos de enseñanza se pueden clasificar en seis familias (Sicilia y Delgado, 2002):

1) Estilos de enseñanza tradicionales, que utilizan una comunicación unidireccional, centrados en el profesor y/o la tarea, alumno dependiente. Tipos:

- Mando directo, se caracteriza por el total protagonismo del profesor. La función del alumno consiste en ejecutar, seguir, obedecer.

- Modificación del mando directo, se diferencia del anterior en que no hay voces de mando, hay más libertad y, por tanto, se dan diferentes formas de colocarse

- Asignación de tareas, lo importante es la tarea que tiene que desarrollar el alumno, la planificación es más flexible y la organización menos rígida.

2) Estilos que fomentan la individualización, la labor del profesor se adapta a las características de los alumnos; aprendizaje individualizado y auto motivado; el alumno interviene en su evaluación y permiten un trabajo sin la presencia del profesor. Tipos:

- Trabajo por grupos, se parte de una evaluación inicial, para conocer niveles o intereses y establecer subgrupos. En función del interés, se 
pueden establecer «contratos didácticos» alumnos-profesor, y los niveles pueden motivar al alumno a progresar de uno a otro.

- Enseñanza modular, conjuga los intereses con el nivel de ejecución.

Se parte de una evaluación inicial, para conocer intereses. Apartir de los módulos de trabajo se determinarán subgrupos por niveles dentro de cada módulo.

- Programa individual, elaboración de un programa tras la evaluación inicial. La información inicial la aportará el profesor de forma escrita o audiovisual.

- Enseñanza programada, hay dos posibilidades: Un programa lineal y los programas ramificados.

3) Estilos que posibilitan la participación, permiten una intervención de los alumnos en el proceso de enseñanza-aprendizaje. Tipos:

- Enseñanza recíproca, se divide la clase por parejas: uno realiza la acción y el compañero observa y da un conocimiento de los resultados.

- Grupos reducidos.

- Microenseñanza, tras una evaluación inicial, se organizan los subgrupos; partiendo del número de alumnos «especialistas» que dominan la materia y quieran asumir la responsabilidad de actuar de profesores. El profesor actúa directamente sobre los alumnos «especialistas».

4) Estilos que implican cognoscitivamente al alumno, tipos:

- Resolución de problemas, más posibles soluciones y menos indicios.

- Descubrimiento guiado, menos soluciones, más ayuda e indicios.

5) Estilos que favorecen la socialización, el profesor da protagonismo al grupo y se apoya en la dinámica del mismo para plantear trabajos de tipo colectivo, donde lo importante no es la ejecución individual sino el trabajo en equipo.

6) Estilos que promueven la creatividad, tienen como objetivo fomentar la diversidad de opinión, facilitar la libre expresión del individuo, impulsar la creación de nuevos movimientos y posibilitar la innovación alumnos-profesor.

Los estilos de enseñanza que utilizan los docentes pueden ser tradicionales o activos. Los estilos tradicionales son construidos sobre la base del entrenamiento deportivo donde los entrenadores basan su planteamiento en la consecución de elementos técnicos individuales, así como sistemas de juego colectivo (Águila y Casimiro, 2002).

El objetivo del presente estudio ha sido estudiar la influencia de los estilos de enseñanza en una sesión de actividad física extraescolar respecto a la motivación y participación que generan en el alumno, para así poder determinar qué estilos favorecen más la motivación intrínseca del alumno y fomentan más su participación.

En cuanto a la forma de enseñar, la metodología de la enseñanza deportiva sugiere la utilización de las distintas técnicas y estilos de enseñanza en función de diferentes variables: edad, experiencia, nivel de capacidad física, grado de motivación intrínseco de los alumnos, personalidad y preferencias del profesor, objetivos y tipo de tarea (Méndez, 1999).

Según Delgado (1999) existe una clara tendencia de los futuros profesores de Educación Física, hacia estilos de enseñanza innovadores, como son los participativos, individualizadores, creativos y socializadores; dejando atrás los estilos tradicionales.

Los objetivos del presente estudio son:

- Determinar la influencia de los estilos de enseñanza en el alumnado de actividades físicas extraescolares en cuanto a su participación y motivación.

- Especificar las diferencias entre los estilos de enseñanza en la calidad de aprendizaje del alumnado de actividad física extraescolar.

- Determinar las posibles modificaciones en la actitud del alumnado hacia la actividad fisica.

\section{Método}

Se plantea un diseño experimental para el análisis de la variable independiente, estilos de enseñanza, en la influencia sobre las variables dependientes, como son motivación, participación y actitud, en clases de educación física en el bloque de contenidos de juegos y deportes.

Los participantes del presente estudio han sido 60 alumnos de Educación Secundaria Obligatoria, estudiantes de $3^{\circ}$ de ESO; con una edad media de 14,300,8 años.

El tipo de selección utilizado para elegir a la muestra objeto de estudio fue un muestreo intencionado $\mathrm{u}$ opinático, dividiendo posteriormente la muestra en dos grupos de 30 sujetos (Grupos A y B).

Los instrumentos utilizados para este estudio han sido dos cuestionarios sobre actitud y satisfacción del alumnado de educación física (Calderón, 2007):

Cuestionario Escala de la actitud del alumno en clase (anexo 1), que pretende valorar la actitud del alumno hacia la práctica de actividad fisica en general. Con este cuestionario se intentó comprobar si existen modificaciones en la actitud del alumno de secundaria hacia la actividad fisica utilizando para su metodología un tipo u otro de estilo de enseñanza.

Cuestionario de satisfacción, participación y opinión de mejora (anexo 2), se utilizará para obtener información sobre el grado de satisfacción en las sesiones de actividad física. El uso de este cuestionario tiene el pretexto de conocer y valorar la satisfacción de los alumnos en las clases de educación física en cuanto a participación, diversión y percepción de mejora que experimentan con las tareas planteadas en las sesiones, comprobando si existen diferencias según usen los docentes un tipo de estilo u otro.

Para la realización de nuestra investigación se ha dividido el conjunto de alumnos en dos grupos experimentales de 15 alumnos cada uno. Al grupo experimental 1 se le aplicó un estilo de enseñanza tradicional (mando directo), mientras que al grupo experimental 2 se le aplicó un estilo de enseñanza activo que fomenta la socialización durante 15 sesiones.

El cuestionario sobre actitud se pasó a los alumnos en la 1a sesión y en la última para así poder comparar datos y observar si hubo progreso o cambio alguno en su actitud y motivación.

\section{Resultados y discusión}

Como podemos apreciar en la figura 1 , nos encontramos con los datos de actitud del alumno antes y después de haber realizado las sesiones. No existen grandes diferencias en su puntuación, ya que el grupo 1, presenta una puntuación de 32 valores antes de las clases y de 34 al finalizar éstas. Por lo que, aunque ha aumentado su valoración en cuanto a la actitud, creemos que no es relevante. Así, se reflejan en los porcentajes de figura 1, donde prácticamente falta más del 50\% para conseguir la mejor actitud posible en ambas situaciones.

El grupo dos (Figura 2) muestra unos datos parecidos en relación al grupo 1, si tenemos en cuenta los datos antes de la intervención en las actividades extraescolares. Así obtenemos valores similares ( 32 grupo 1 y 31 grupo 2), y se refleja en un $45 \%$ de actitud frente a la práctica de actividades extraescolares.
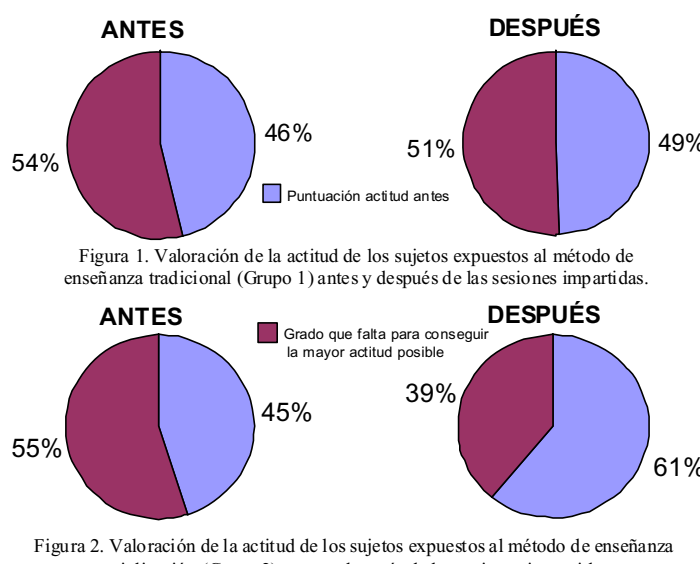
socialización (Grupo 2) antes y después de las sesiones impartidas. 
Sin embargo, en este grupo (grupo 2) encontramos una mejoría más notable en relación a los datos obtenidos antes de la intervención. Así podemos observar (Figura 2) una mejoría en la actitud de 16 puntos porcentuales. Lo que nos indica que este estilo de enseñanza cosecha una mejor actitud por parte del alumno hacia la realización de la actividad extraescolar. Además, si lo comparamos con el otro grupo (grupo 1) vemos como esta mejoría es mucho mayor, ya que, aunque los dos muestran mejores valores después de las sesiones, el grupo dos mejoró 13 puntos más que el grupo 1.

En relación a la satisfacción, participación y opinión de mejora del alumno antes y después de las sesiones, obtenemos 13 puntos en el grupo 1 , antes de la intervención y una sutil mejoría ( 2 puntos) cuando estos contenidos son preguntados después de la intervención didáctica. Así, apreciamos que el sólo el $41 \%$ manifiesta una buena satisfacción al inicio de las actividades. Sin embargo, tras la aplicación didáctica, podemos ver ahora una mayor mejoría que en la actitud, ya que se aumenta 6 puntos porcentuales el nivel de satisfacción frente a los 3 que encontrábamos en relación a la actitud. De todos modos, en ambos casos, no se supera el $50 \%$.

Al igual que ocurría en relación a la actitud, obtenemos datos muy similares entre el grupo 1 y el grupo 2 antes de comenzar la intervención didáctica (13 y 14 valores respectivamente). Sin embargo, y con más incremento que el hallado en la actitud, cuando se utiliza un estilo de enseñanza que propicia la socialización, vemos como aumenta (Figura 4) de una forma importante. Si lo comparamos con el grupo 1, nuevamente vemos como el aumento mayoritario se da en el grupo 2 (estilo de enseñanza socializador), encontrándose una diferencia entre los dos grupos de 10 valores, lo que equivale a 31 punto porcentual. Podemos deducir, por tanto, que este estilo de enseñanza aumenta considerablemente el nivel de satisfacción, participación, y opinión de mejora del alumno.

Así mientras en el grupo 1 se vio un incremento en la satisfacción, participación, y opinión de mejora del $6 \%$, en el grupo 2 se vio un incremento del $34 \%$. Por tanto se ve una mayor mejoría con el método de enseñanza activo basado en la socialización.

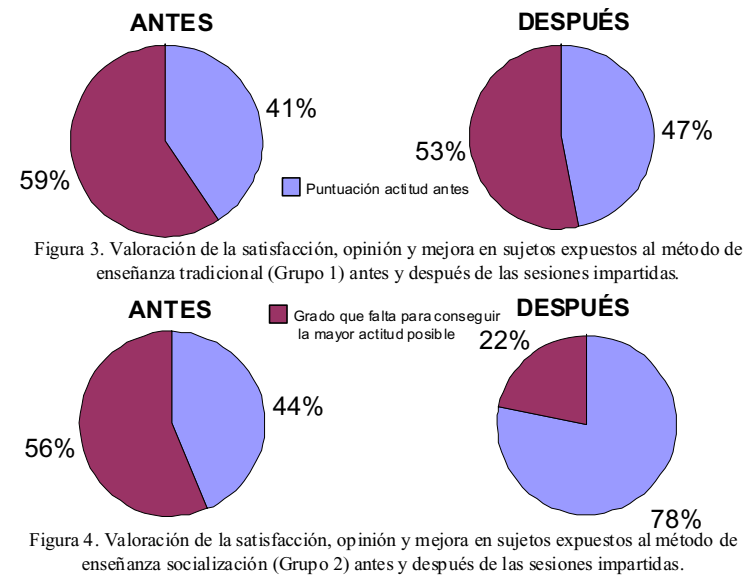

\section{Conclusiones}

En base a los datos encontrados podemos concluir que:

- Los métodos de enseñanza tradicional y socialización casi no modifican la actitud del alumno hacia la tarea.

- El método tradicional y socializador influyen positivamente en la satisfacción del alumno.

- La satisfacción tiene un mayor aumento que la actitud cuando se aplica un método de enseñanza al alumno.

Ninguno de los estilos de enseñanza provoca un descenso de actitud o satisfacción en el alumno.

\section{Bibliografía}

Águila, C., Casimiro, A. (2002). Consideraciones metodológicas para la enseñanza des deportes colectivos en edad escolar. Ef deportes. Extraído el 11 de Diciembre, 2007 de http://www.efdeportes.com/efd17a/ motiv.htm.

Calderón,A. (2007). Incidencia de la forma de organización sobre la cantidad y la calidad de la práctica, el feedback impartido, la percepción de satisfacción, y el aprendizaje en la enseñanza de habilidades atléticas. (Tesis doctoral). Universidad Católica San Antonio.

García, M. (2005). Encuesta sobre hábitos deportivos de los españoles. Avance de resultados. Madrid: Consejo Superior de Deportes.

Delgado, M.A. (1999). Comparación de la valoración de los estilos de enseñanza por futuros profesores de ecuación física durante la formación inicial y profesores de educación física en formación permanente. Ef deportes. Extraído el 10 de Diciembre, 2007 de http:// www.efdeportes.com/efd17a/motiv.htm.

Gómez, M., Ruiz, F., García, M., y Piéron, M. (2003). Percepción de disponibilidad de tiempo libre, un estudio longitudinal en educación secundaria post obligatoria. In F. Ruiz y E. P. González (Eds.), Educación Física y Deporte en Edad escolar. Actas del V Congreso Internacional de FEADEF (pp.537-544). Valladolid: Federación Española de Asociaciones de Docentes de Educación Física [FEADEF].

Méndez, A. (1999). Modelo de Enseñanza deportiva. Análisis de dos décadas de investigación. Ef deportes. Extraído el 20 de Noviembre, $2007 \mathrm{de} \mathrm{http://www.efdeportes.com/efd17a/motiv.htm.}$

Moreno, J.A. y Hellín, M. G. (2007), 2 (9). El interés del alumnado de Educación Secundaria Obligatoria hacia la Educación Física, Revista Electrónica de Investigación Educativa, 9(2). Extraído el 20 de Noviembre, $2007 \mathrm{de} \mathrm{http://redie.uabc.mx/vol9no2/contenido-moreno.html.}$

Ruiz, F., y García, M. (2002). Práctica de actividad físico deportiva de tiempo libre y motivos para realizarla. Estudio comparativo entre el alumnado de enseñanza secundaria postobligatoria y de segundo ciclo de la Universidad de Almería. Efdeportes. Extraído el 10 de Diciembre, 2007 de http://www.efdeportes.com/efd17a/motiv.htm.

Sáenz, P., Ibáñez, S., y Jiménez, F. (2000). La motivación en clase de educación física. Ef deportes. Extraído el 10 de Diciembre, 2007 de http://www.efdeportes.com/efd17a/motiv.htm.

Sicilia, A. y Delgado, M. A. (2002). Educación Física y estilos de enseñanza: Análisis de la participación del alumnado desde un modelo socio-cultural del conocimiento escolar. (P. 34-96). Barcelona: Inde. 